Case Report:

\title{
A Giant Abdominal Cerebrospinal Fluid Pseudocyst
}

\author{
Mohamed Ouladsaiad ${ }^{1,2 *}$, Hamza Hokoumi ${ }^{1,2}$, Najoua Aballa ${ }^{1,2}$
}

1. Department of General Pediatric Surgery, School of Medicine, Cadi Ayyad University, Marrakesh, Morocco

2. Mother and Child Unit, Mohammed VI University Hospital, Marrakesh, Morocco

Citation: Ouladsaiad M, Hokoumi H, Aballa N. A Giant Abdominal Cerebrospinal Fluid Pseudocyst. Iran J Neurosurg. 2017

3(3):109-114. https://doi.org/10.29252/IRJNS.3.3.109

: https://doi.org/10.29252/IRJNS.3.3.109

Article info:

Received: 18 June 2017

Last Revised: 26 July 2017

Accepted: 13 September 2017

Keywords:

Ventriculoperitoneal shunt, Abdominal, Pseudocyst,

Cerebrospinal fluid,

Hydrocephalus, Encephalocele

\section{ABSTRACT}

Background and Aim: Ventriculoperitoneal shunt (VPS) is the most commonly used procedure for treatment of hydrocephalus in children. Abdominal cerebrospinal fluid pseudocyst is a rare complication but potentially fatal. The pathogenesis of this complication remains unclear. This complication should be considered during differential diagnosis of an abdominal mass. Our aim is to report how large and giant an abdominal cerebrospinal fluid pseudocyst can develop, and how severe the neurological damage can be.

Case Presentation: A 6 year-old girl known to have a ventriculoperitoneal shunt presented with abdominal distension and signs of increased Intracranial Pressure (ICP). Ultrasonography revealed a hypoechoic intraperitoneal fluid containing cyst with a well-defined margin and septations. Abdominal computerized tomography revealed a huge cyst in the abdomen and the distal part of the peritoneal shunt tube located within the cyst. Laparotomy revealed a huge cyst extending from the upper right side of the abdomen to the pelvis. The whole cyst was excised and the catheter repositioned.

Conclusion: Early diagnosis of abdominal Cerebrospinal Fluid (CSF) pseudocyst is only possible through close and periodic examination of patients after the procedure.

\section{Background and Importance}

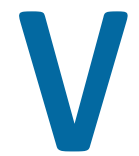

entriculoperitoneal Shunt (VPS) is the most commonly used procedure to relieve hydrocephalus. Extracranial complications that are associated with the distal segment of the peritoneal shunt are infection, omental clogging, abdominal visceral perforation, bowel obstruction, extra peritoneal retraction of the catheter, development of incisional hernia, abdominal CSF containing pseudocyst and subcutaneous collection of CSF $[1,2]$. Abdominal CSF Pseudocyst (ACP) is characterized by a fluid filled collection of CSF in the peritoneal cavity containing the distal end of the VPS catheter and is surrounded by a wall composed of fibrous tissues without an epithelial lining [1].

ACP was first described by Harsh in 1954 and its incidence ranges from less than $0,33 \%$ to $6,8 \%[1,3]$. We report the hugest abdominal cerebrospinal fluid pseu-

* Corresponding Author:

Mohamed Ouladsaiad, PhD

Address: Department of General Pediatric Surgery, School of Medicine, Cadi Ayyad University, Marrakesh, Morocco

Tel: +98 (21) 2673083983

E-mail: mouladsaiad@gmail.com 
docyst in children ever reported in the literature presenting with advanced neurological deficit (Table 1) [4-6].

\section{Case Presentation}

A 6-year-old girl was admitted to our emergency center in Mohammed VI University Hospital for progressive abdominal distension and headache that was witnessed by her mother from two years before that. A VPS was placed at the age of three months to treat hydrocephalus secondary to surgery of occipital encephalocele. No other abdominal surgery had been performed before or after this VPS.

First of all, a written informed consent was obtained from the patient's parents. Physical examination revealed a diffuse abdominal distension as shown in Figure 1 and signs of high intracranial pressure were present: headache and sunset eyes sign, without fever. Plain X- ray of abdomen had shown an abdominal distension with mass effect on the center and left side pushing bowel loops towards the right lower quadrant with no free air in the peritoneal cavity. We noticed the distal tip of the ventriculoperitoneal shunt tube in the right lumbar region as you can see in Figure 2. Abdominal ultrasound examination revealed a large anechoic loculated intraperitoneal collection with few internal septations and peritoneal end of shunt catheter within the cyst. Abdominal CT scan with contrast medium revealed a huge cystic mass of $11.8 \times 15.6 \times 30$ cubic $\mathrm{cm}$, occupying most of the abdominopelvic cavity around the distal tip of the ventriculoperitoneal shunt tube. It was thin-walled with internal septations that did not enhance after contrast medium administration. This huge mass pushed forward the abdominal wall and displaced the bowel loops and adjacent organs backward as it is shown in Figure 3a. Head computed tomography with contrast medium revealed a major hydrocephalus of the third and lateral ventricles, compressing and thinning the overlying cortex without features of periventricular oozing. Meanwhile, the proximal tip of the shunt was located within lateral ventricles (Figure $3 b$ ). The treatment consisted of laparotomy with total cyst excision (Figure 4) and catheter repositioning. Microbiological analysis of CSF from the pseudocyst showed no evidence of infection. Histological analysis revealed a thin-walled cystic mass. The wall composed of fibrous tissue without an epithelial lining. Numerous small vessels can be noticed, with the absence of any significant inflammatory infiltrate. The border was filled with cerebrospinal debris. After a follow-up of six months, the patient was doing well with a noticed improvement in her motor skill, walking, and speech.

\section{Discussion}

VPS is the most frequently used procedure for hydrocephalus $[7,8,10]$. Complications associated with the peritoneal tip of the VPS include viscera perforation, migration of the shunt, bowel obstruction secondary to adhesions, metastatic spread via the shunt and abdominal cerebrospinal fluid pseudocyst. The latest

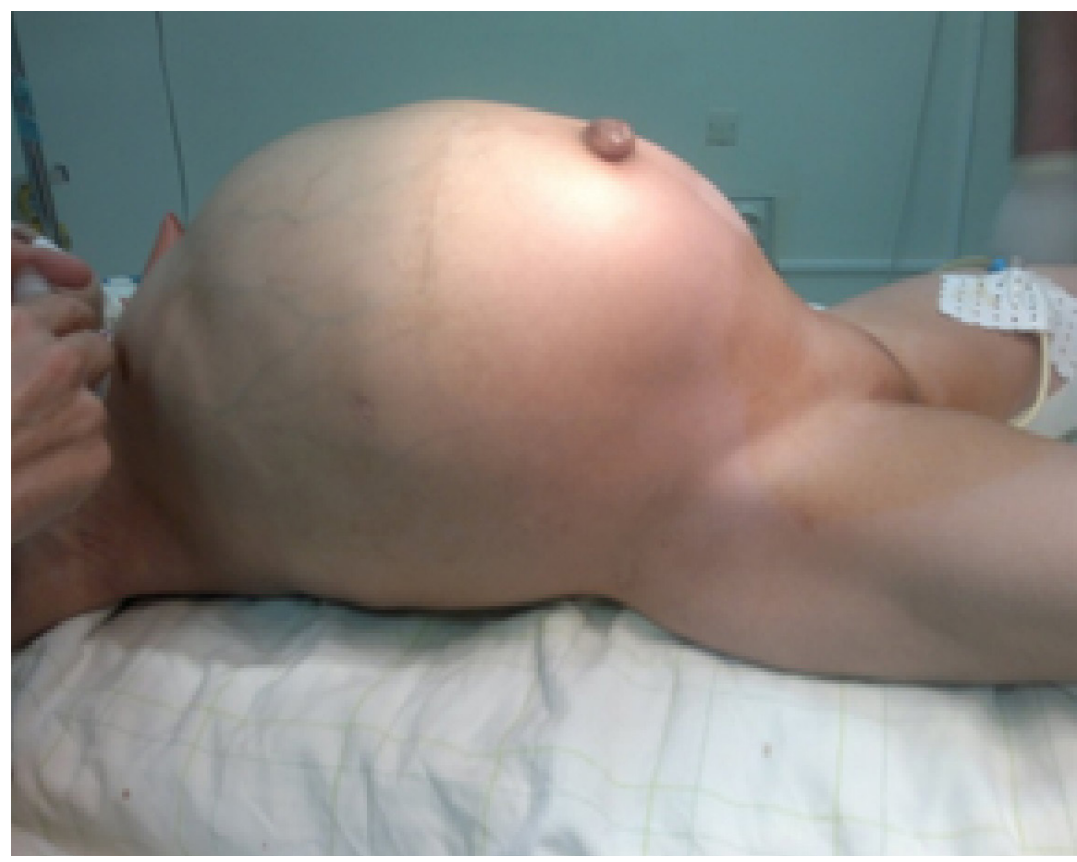

Figure 1. Huge abdominal distension 


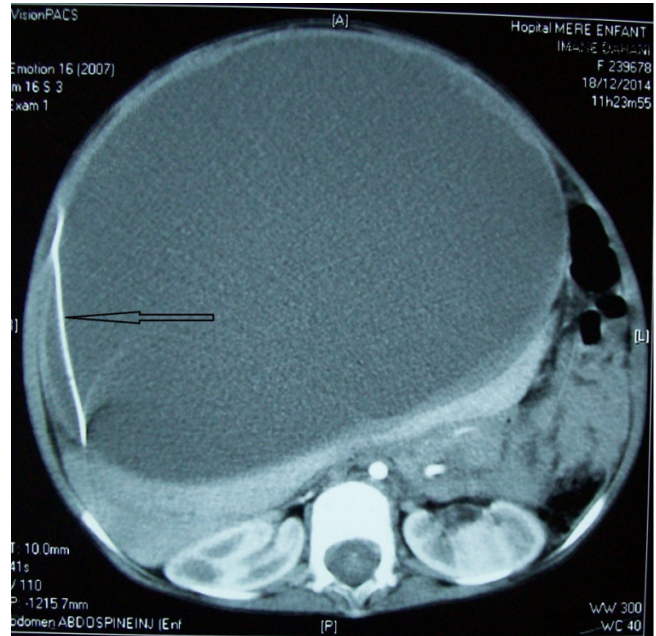

a

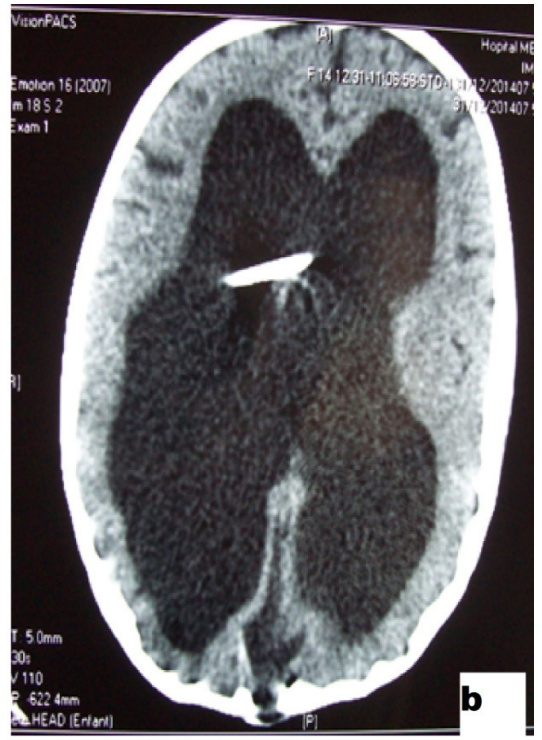

No

Figure 2. Abdominal RX (arrow) showing distal tip of the ventriculo-peritoneal shunt tube

complication is rare but potentially life-threatening [11]. ACP is a collection of CSF around the shunt tip in the peritoneal cavity. The wall of pseudocyst is composed of fibrous tissues without an epithelial lining [12]. The physiopathological mechanism of pseudocyst remains unclear. However, several predisposing factors for cyst formation have been reported including prior surgical peritoneal adhesions, distal shunt migration, multiple shunt revisions, allergic reactions increase in the protein content of CSF and inflammatory processes in the peritoneum, either sterile or infectious $[12,14]$.

Staphylococcus epidermis and Staphylococcus aureus are the most commonly detected bacteria [11-

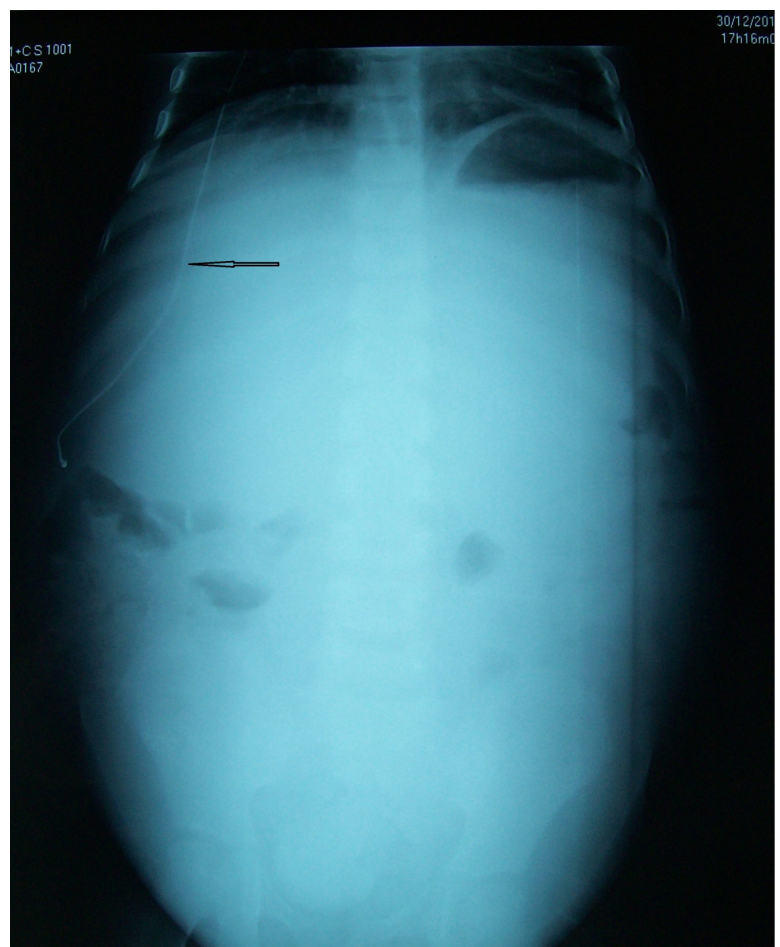

Figure 3. a- Abdominal CT (arrow) showing the distal tip of ventriculo shunt tube within the hugecyst. b- Head CT major hydrocephalus compressing and thining the overlying cortex 


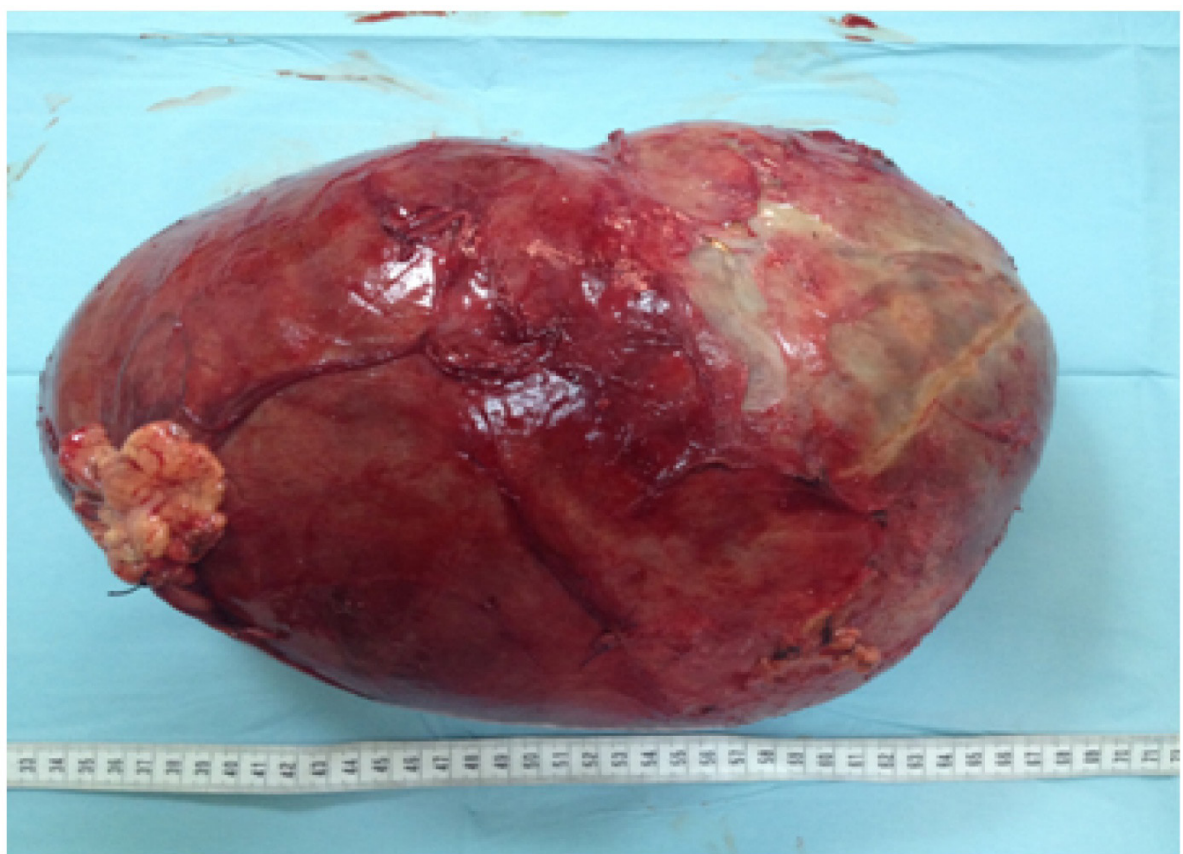

Figure 4. The giant abdominal cyst totally removed

14]. The peritoneum is not the only site of collection formation; cases with CSF collections have also been reported in subcutaneous cellular tissue, in the breast after breakage and migration of the distal portion of the catheter, in the neck because of perforation of the shunt after jugular catheterization and the liver is another unusual site of pseudocyst formation [15]. Children with ACP typically present with symptoms of shunt malfunction such as headache, nausea and vomiting as opposite to adult patients who present with abdominal signs [1-11]. In the present report, signs of high intracranial pressure were the main complaints for referral. Plain radiographs are often normal, but they might show the continuity of the shunt tube in a soft tissue mass that displaces adjacent bowel loops [16]. Ultrasonography demonstrates the presence of localized peritoneal fluids collection visualized as a smooth pseudomembrane echo-free fluid collection with or without septations and its relation to the catheter [16].

Table 1. Size of cerebrospinal fluid pseudocyst reported by different authors

\begin{tabular}{ccc}
\hline Authors & Age (Years) & Size of the Cyst (cm) \\
\hline & 58 & $4 \times 4$ \\
& 34 & $20 \times 13$ \\
F Aparici-Robles [4] (n=6) & 35 & $4 \times 2 \times 15$ \\
& 22 & $19 \times 12$ \\
Stetson RC [5] $(n=1)$ & 26 & $3 \times 4$ \\
Chung-Hsin Lee [6] $(n=1)$ & 44 & $29 \times 18 \times 14$ \\
Our case & 14 & $28 \mathrm{~cm} \times 25 \times 13$ \\
\hline
\end{tabular}


Computerized tomography is considered effective in the definitive diagnosis of ACP when they are large and deform the normal architecture of the abdomen [1] such as in our case. Diagnosis can also be reached with nuclear shuntograms after radioisotope injection into the VPS $[4,16]$. Differential diagnosis of an abdominal CSF pseudocyst includes lymphangioma, mesenteric cyst, pancreatic pseudocyst, duplication and cystic teratoma. When an ACP is diagnosed it is important to scan the brain for increased intracranial pressure as in our case. The presence or absence of infection is a key factor in making a definitive decision about when and how surgical treatment should be performed. Several surgical options can be considered such as: laparotomy with wide excision of cystic wall, paracentesis and aspiration of cystic fluid, repositioning the distal peritoneal catheter in a different abdominal quadrant, repositioning of the peritoneal catheter in retrohepatic space, external ventricular drainage, conversion of the system to either ventriculo-atrial or ventriculopleural shunt, and endoscopic third ventriculostomy in selected cases $[15,16]$.

Recently laparoscopic management has also been reported [17]. If infection is confirmed, the shunt is externalized and the appropriate antibiotic treatment must be used. A new shunt placement should be performed after improvement of the abdominal symptoms and infection [14-16]. In the absence of infection, excision of the cyst walls and reposition of the catheter within the peritoneal cavity [15]. Anecdotal reports about spontaneous resorption of the cyst without excision or aspiration after removal of peritoneal catheter [16]. If only the abdominal CSF pseudocyst becomes symptomatic, it may be drained percutaneously while awaiting elective revision of the shunt [16].

\section{Conclusion}

Pediatric surgeon, gastroenterological surgeons and pediatrician should be aware of ACP during differential diagnosis of an abdominal mass. Early diagnosis by close clinical examination would improve the outcome of VPS and reduce patient's neurological damage.

\section{Acknowledgments}

This research did not receive any specific grant from funding agencies in the public, commercial, or not-forprofit sectors.

\section{Conflict of Interest}

The authors declare no conflicts of interest. Authors' contribution is as follows: Conception and Design: All authors; Data Collection: Mohamed Ouladsaiad, Hamza Hokoumi, Najoua Aballa; Drafting the Article: Mohamed Ouladsaiad, Hamza Hokoumi, Najoua Aballa; Critically Revising the Article: Mohamed Ouladsaiad; Reviewing Submitted Version of Manuscript: Mohamed Ouladsaiad; and Approving the Final Version of the Manuscript: All Authors.

\section{References}

[1] Sena FG, Sousa RM, Meguins LC. Abdominal cerebrospinal fluid pseudocyst: a complication of ventriculoperitoneal shunt in a Brazilian Amazon Woman. Case report. Il Giornale Di Chirurgia. 2010; 31(8-9):371-3. PMID: 20843439

[2] Leung GKK. Abdominal cerebrospinal fluid (CSF) pseudocyst presented with inferior vena caval obstruction and hydronephrosis. Child's Nervous System. 2010; 26(9):1243-5. doi: 10.1007/s00381-010-1221-z

[3] Pathi R, Sage M, Slavotinek J, Hanieh A. Abdominal cerebrospinal fluid pseudocyst. Australasian Radiology. 2004; 48(1):61-3. doi: 10.1111/j.1440-1673.2004.01245.x

[4] Aparici-Robles F, Molina-Fabrega R. Abdominal cerebrospinal fluid pseudocyst: a complication of ventriculoperitoneal shunts in adults. Journal of Medical Imaging and Radiation Oncology. 2008; 52(1):40-3. doi: 10.1111/j.14401673.2007.01909.x

[5] Stetson RC, Goyal KA, Sandefur BJ. Abdominal cerebrospinal fluid pseudocyst masquerading as ascites in an adolescent girl. The Journal of Emergency Medicine. 2017, 52(4):e159-e61. doi: 10.1016/j.jemermed.2016.11.021

[6] Lee CH, Cheng WY, Shen CC. Large pseudocyst in the anterior extraperitoneal space as a complication of ventriculoperitoneal shunt. Formosan Journal of Surgery. 2015; 48(4):130-2 doi: 10.1016/j.fjs.2015.03.002

[7] Grosfeld JL, Cooney DR, Smith J, Campbell RL. intraabdominal complications following ventriculoperitoneal shunt procedures. Pediatrics. 1974; 54(6):791-6. PMID: 4431676

[8] Arnell K, Olsen L. Distal catheter obstruction from noninfectious cause in ventriculo-Peritoneal shunted children European Journal of Pediatric Surgery. 2004; 14(4):245-49. doi: $10.1055 /$ s-2004-817896

[9] Pernas JC, Catala J. Case 72: Pseudocyst around Ventriculoperitoneal Shunt. Radiology. 2004; 232(1):239-43. doi: 10.1148/ radiol. 2321011976

[10] Beluffi G, Facchinetti C, Zacchino M. Acute hydrocephalus caused by an abdominal cerebro-spinal fluid pseudocyst around the tip of a ventriculo-peritoneal shunt. European Journal of Radiology Extra. 2006; 59(3):97-9. doi: 10.1016/j. ejrex.2006.06.003 
[11] Tamura A, Shida D, Tsutsumi K. Abdominal cerebrospinal fluid pseudocyst occurring 21 years after ventriculoperitoneal shunt placement: A case report. BMC Surgery. 2013;13(1):27. doi: $10.1186 / 1471-2482-13-27$

[12] Mobley LW 3rd, Doran SE, Hellbush LC. Abdominal pseudocyst predisposing factors and treatment algorithm. Pediatric Neurosurgery. 2005; 41(2):77-83. doi: 10.1159/000085160

[13] Hashimoto M, Yokota A, Urasaki E, Tsujigami S, Shimono M. A case of abdominal CSF pseudocyst associated with silicone allergy. Child's Nervous System. 2004; 20(10):761-64. doi: 10.1007/s00381-003-0904-0

[14] Ohba S, Kinoshita Y, Tsutsui M, Nakagawa T, Shimizu K, Takahashi $\mathrm{T}$ et al. Formation of abdominal cerebrospinal fluid pseudocyst. Neurologia Medico-chirurgica. 2012; 52(11):83842. doi: $10.2176 /$ nmc. 52.838

[15] Santos de Oliveira R, Barbosa A, Vicente YA de MV de, Rubens Machado H. An alternative approach for management of abdominal cerebrospinal fluid pseudocysts in children. Child's Nervous System. 2006; 23(1):85-90. doi: 10.1007/s00381-006-0183-7

[16] Vassilyadi M, Yuh SJ. Management of abdominal pseudocyst in shunt-dependent hydrocephalus. Surgical Neurology International. 2012; 3(1):146. doi: 10.4103/2152-7806.103890

[17] Popa F, Grigorean VT, Onose G,Popescu M, Strambu V, Sandu AM. Laparoscopic treatment of abdominal complications following ventriculoperitoneal shunt. Journal of Medicine and Life. 2009; 2(4):426-36. PMID: 20108757 